\section{Fraser Syndrome: A Case Report of a Very Rare Syndrome}

\section{Abstract}

Fraser syndrome is a rare autosomal recessive multiple malformation syndrome. It is characterized by cryptophthalmos, cutaneus syndactyly, malformation of the larynx and genitourinary tract, craniofacial dysmorphisms, mental retardation, and musculoskeletal abnormalities. The diagnosis of this syndrome can be made on the basis of physical examination and occasionally with prenatal ultrasound imaging. Various mutations of FRAS1, FREM2 and GRIP1 genes are known to play a role in the etiology of the disorder. In this article we want to introduce a case of a female newborn, who presented with cryptophthalmos, syndactyly of both feet and hands, ambiguous genitalia, anal fistule, umbilical hernia, unilateral renal agenesis, and craniofacial dysmorphism. In this case report, we aimed to present and discuss our patient's clinical findings, genetic basis of Fraser syndrome and follow-up cases from the literature.

Keywords: Fraser syndrome; Cryptophthalmos; Syndactyly; Ambiguous genitalia

Received: October 07, 2019; Accepted: November 11, 2019; Published: November 18,2019

\section{Introduction}

Fraser Syndrome (FS) is a rare autosomal recessive disorder. Its estimated frequency is 0.49 per 100,000 lives born [1]. Fraser syndrome is characterized by variable expression of cryptophthalmos, cutaneous syndactyly, dysmorphic ears and nose, laryngeal stenosis, renal and genital abnormalities (bilateral or unilateral renal agenesis, ambiguous genitalia), orofacial clefts, skeletal defects, umbilical hernia and mental retardation. Cryptophthalmos is the most important diagnostic feature of FS and is present in $90 \%$ of affected patients $[1,2]$. Laryngeal stenosis is a common cause of death. Prognosis is poor and depends on the dominant pathology. In this report we presented a case of a patient with rare Fraser syndrome. Our patient has multiple organ anomalies, but no fatal ones, like laryngeal malformation or bilateral renal agenesis. The aim of our study is to discuss this case and to show the viability of the affected patient due to phenotype variability of genetic mutations associated with Fraser syndrome.

\section{Case Report}

A 32-year-old woman (gravida 2 and parity 2) delivered a female baby with caesarean section in the $38^{\text {th }}$ week of pregnancy. This was the second child of the second-degree consanguineous

\section{Olena Erkun ${ }^{1 *}$, Tetyana Syrotenko², Ibrahim Caner ${ }^{3}$, Oner Ozdemir ${ }^{4}$, Mine Urfali ${ }^{5}$, Tugba Akin Duman ${ }^{6}$ and Yasemin Anil Eyupoglu Tanriverdi ${ }^{5}$}

1 Department of Pediatrics, Sakarya University Research and Training Hospital, Sakarya, Turkey

2 Department of Medicine, Marmara University, Istanbul, Turkey

3 Department of Pediatrics, Division of Neonatology, Sakarya University Faculty of Medicine, Sakarya University Research and Training Hospital, Sakarya, Turkey

4 Department of Pediatrics, Division of Allergy and Immunology, Sakarya University Faculty of Medicine, Sakarya University Research and Training Hospital, Sakarya, Turkey

5 Department of Medical Genetics, Sakarya University Research and Training Hospital, Sakarya, Turkey

6 Department of Medical Genetics, Haseki Research and Training Hospital, Istanbul, Turkey

\section{*Corresponding author: Olena Erkun}

Department of Pediatrics, Sakarya University Research and Training Hospital, Sakarya, Turkey.

” elena.baluka@gmail.com

Tel: +905394851947

Citation: Erkun O, Syrotenko T, Caner I, Ozdemir O, Urfali M, et al. (2019) Fraser Syndrome: A Case Report of a Very Rare Syndrome. J Rare Disord Diagn Ther. Vol.5 No.3:5

marriage. According to the parents of this baby, their first child had the same abnormalities in his appearance upon the birth and 
died within first 30 minutes of life. The baby's weight was 2,910 g (25-50 percentile), height $46 \mathrm{~cm}$ (10-25 percentile) and head circumference $34 \mathrm{~cm}$ (50-75 percentile), heart rate $>100 / \mathrm{min}$, Apgar score was 8-9. The baby cried immediately after birth. The physical examination has shown complete cryptophthalmos of the left eye and partial cryptophthalmos of the right eye, flat nose with wide nasal bridge, hypertelorism, low position of hair growth line of the forehead (Figure 1), high palate, bilateral hypoplasia of external ears, widely located nipples, ambiguous genitalia (clitoromegaly, hypoplasia of labia major and minor), perianal fistula, and umbilical hernia. Additionally, complete cutaneous syndactyly of both hands and feet (Figures $\mathbf{2}$ and $\mathbf{3}$ ) was observed. There were no signs of tracheal stenosis or cardiovascular system abnormality. On the abdominal ultrasonography, right kidney size and parenchymal thickness were normal. No mass, calculus, or hydronephrosis were found. Left renal agenesis with normal urinary bladder was detected. Findings of bicornuate uterus were observed. No other pathology was observed. No laryngomalacia and laryngostenosis were detected. By using Next Generation Sequencing platform, we detected a homozygous FRAS1 gene mutation in exon 26, c.3730 C>T (p.Arg1244Ter) ( $r$ 186964660) mutation clinical significance was considered pathogenic from mutation databases (Human Gene Mutation Database (HGMD) Professional, Varsome) (Figure 4). The same mutation was found in heterozygous form in the parents. Sanger confirmation was performed in all the individuals who had the mutation. (Informed consent was obtained from the parents of the patient, presented in this report.)

\section{Discussion}

Fraser syndrome is characterized by cryptophthalmos, syndactyly, renal defects, ambiguous genitalia, anus defects, larynx and trachea anomalies, dysplastic ears, frontal and nasal bone defects, umbilical and nasal anomalies [1,2]. FS is diagnosed according to major and minor criteria. Major criteria are the presence of cryptophthalmos, syndactyly, abnormal genitalia and affected sibling with FS. Minor criteria are congenital malformation of nose, ears, larynx, cleft lip and palate, skeletal defects, umbilical hernia, renal agenesis, and mental retardation

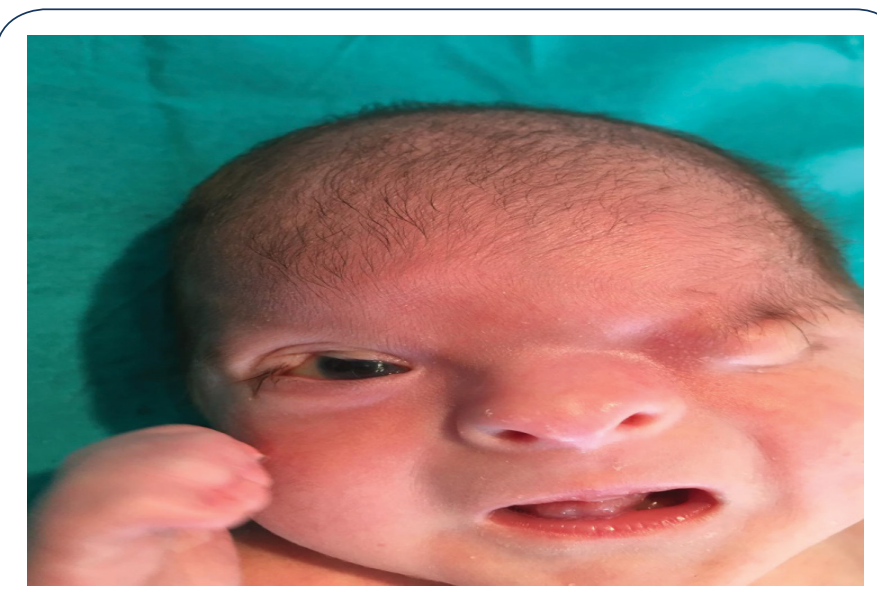

Figure 1 Cryptophthalmos, hypertelorism and flattened-wide nasal root in the right eye.

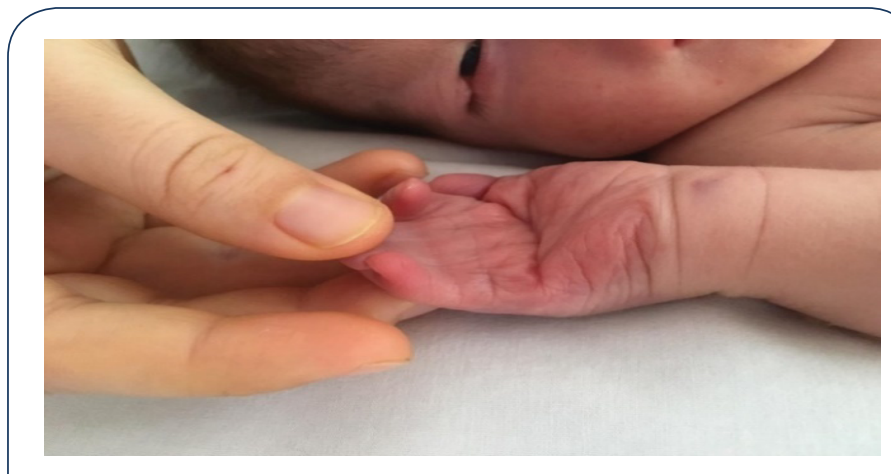

Figure 2 Syndactyly of the upper extremities.

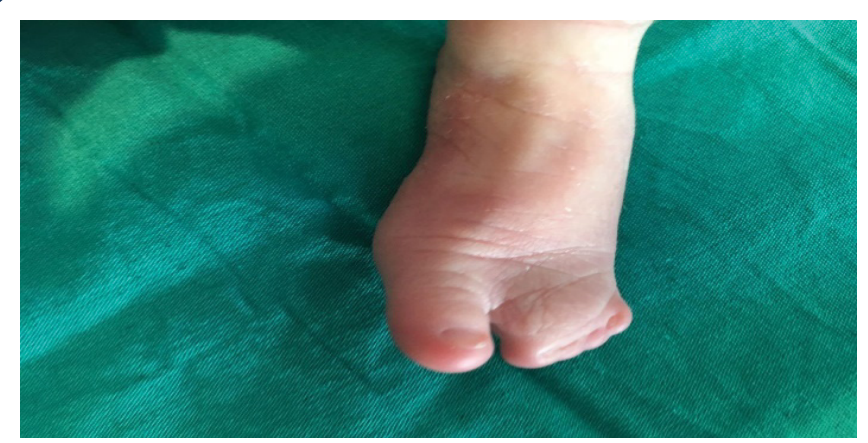

Figure 3 Syndactyly of the lower extremities.

[1]. Three major, or two major and two minor, or one major and three minor criteria should be met for the diagnosis of Fraser syndrome [1]. The definitive diagnosis of the disorder is made by demonstrating FRAS1 (4q21) and FREM2 (13q13.3), GRIP1(12q14.3) gene mutations [3-6]. Proteins, which are encoded by these genes, play a role in adhesion of epidermis to the basal membrane. FS gene mutations are responsible for the structures formed under epidermis during embryonic life $[4,5]$. In the literature, cryptophthalmos is the most common finding in FS. Deficient formation of the eyelids may lead to bilateral, complete, partial, abortive variants of cryptophthalmos $[1,2]$. In our case, complete left and partial right cryptophthalmos is seen. Syndactyly is the most common anomaly of extremities in FS $[1,2]$. In our case, cutaneous syndactyly of both hands and feet was found. The FS patients usually have laryngeal anomalies as well, which frequently lead to death during early postnatal period. Our patient was not diagnosed with any laryngeal anomalies. In the literature, imperforated anus, anal atresia, stenosis, perianal fistula, umbilical hernia, bilateral and unilateral renal agenesis and ambiguous genitalia have also been found in the FS patients $[1,2,6]$. In our case, 3 major (cryptophthalmos, syndactyly, ambiguous genitalia) and 4 minor (anal fistula, hypoplastic nose, umbilical hernia, unilateral renal agenesis) criteria for the diagnosis were met. Most patients with Fraser syndrome die within the first week of their life. Life span varies depending on the presence of fatal anomalies, such as laryngeal stenosis, atresia, or bilateral renal agenesis $[3,7]$, which were not found in our case. Mutations in FRAS1, FREM2, and GRIP1 genes have been identified causing FS. Mutation in 


\section{A}

\begin{tabular}{|c|c|c|c|c|c|c|c|c|c|c|c|c|c|}
\hline $\mathbf{G}$ & 吾 & 드. & 吾 & $T$ & C & $T$ & $G$ & 吾 & $G$ & $\underline{n}$ & $T$ & $G$ & . \\
\hline$G$ & 吾 & 드. & 吾 & $\mathrm{T}$ & C & $\mathrm{T}$ & $G$ & 吾 & $G$ & 吾 & $T$ & $G$ & 吾 \\
\hline
\end{tabular}

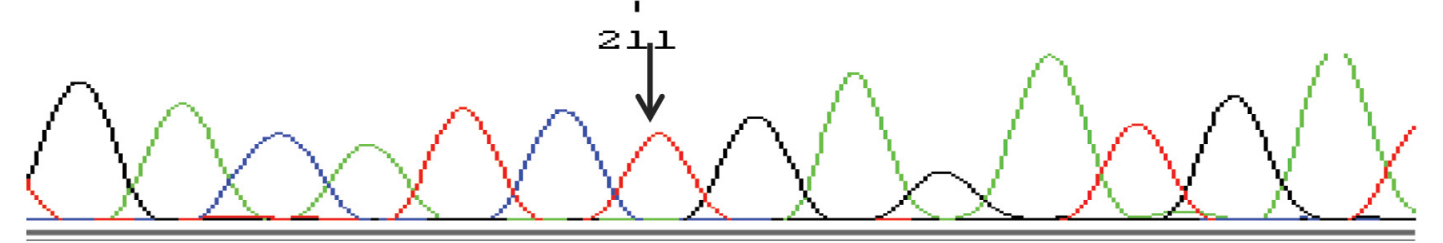

B

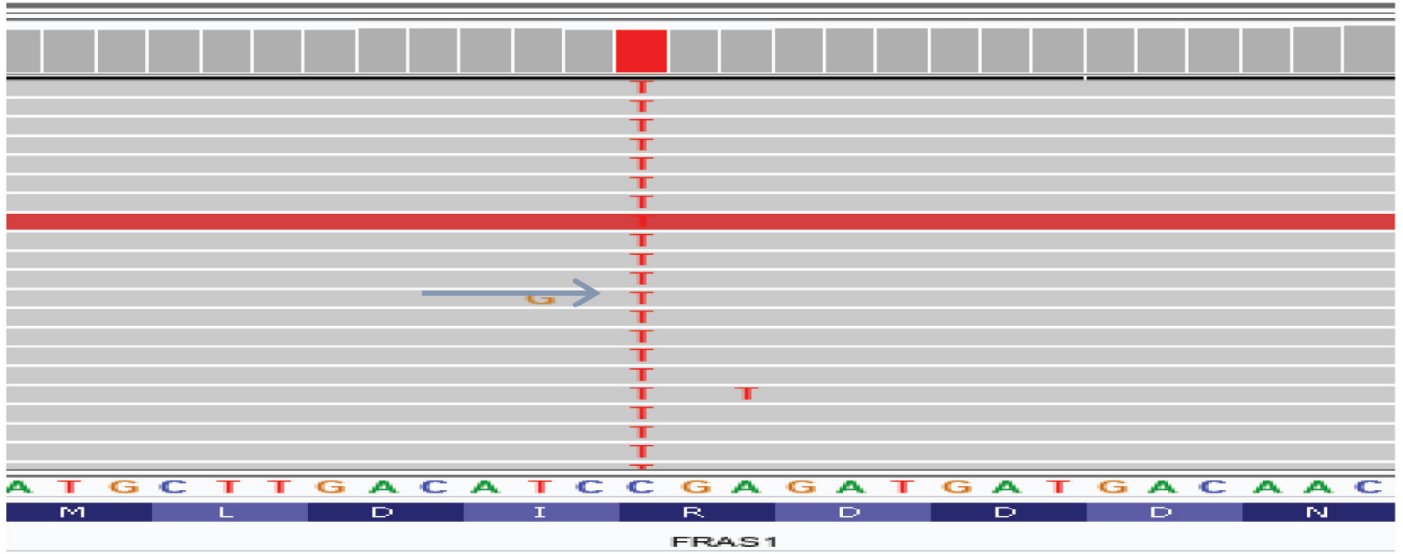

Figure 4 FRAS1 gene mutation images of the patient. A: Black arrow shows homozygous p.Arg1244Ter mutation in FRAS gene by Sanger sequencing. B: Blue arrow shows homozygous p.Arg1244Ter mutation in FRAS gene by next-generation sequencing (NGS).

FRAS1 is responsible for the classical phenotype of FS. The FRAS1 gene is located on chromosome $4 \mathrm{q} 21.21$ and encodes a protein that is widely expressed. Nucleotide substitutions, splicing defects, large insertion and small deletions/insertions have been described to be responsible for FS. 61 mutations are listed in HGMD; most of them are truncating [4-6]. However, mutation analysis cannot be performed in most cases described in the literature, and since many patients are lost during the prenatal and perinatal periods, the diagnosis is often made based on the clinical findings. If mutation is known, Preimplantation Genetic Diagnosis (PGD) and prenatal diagnosis can be made from fetus sample. There is no specific treatment for the syndrome, only symptomatic treatments can be performed. According to the findings, oculoplasty, corneal surgery, genital reconstruction can be performed. The patient is now 8 months old, weight is $9 \mathrm{~kg}$, height $67 \mathrm{~cm}$, follow-up continues [7,8].

\section{Conclusion}

In summary, the patient was diagnosed with Fraser syndrome based on anamnesis (the history of sibling born with the same appearance abnormalities), clinical findings assessed according to the criteria (3 major: cryptophthalmos, syndactyly, ambiguous genitalia, and 4 minor: anal fistula, hypoplastic nose, umbilical

\section{References}

1 Slavotinek AM, Tifft CJ (2002) Fraser syndrome and cryptophthalmos: A review of the diagnostic criteria and evidence for phenotypic hernia, unilateral renal agenesis), and genetic test results (positive FRAS1 mutation). Since FS is inherited by autosomal recessive pattern and all parents keep heterozygous mutation, the family has a $25 \%$ chance of having a child with FS again. Genetic counselling was given to the patient's family. It was emphasized that if they had spontaneous pregnancy, prenatal diagnosis of mutation in FRAS1 gene could be made from fetus sample, or they can prefer PGD with in vitro Fertilization (IVF) to have healthy children.

1. Fraser syndrome is an autosomal recessive disease that may affect a child in consanguineous family with each parent carrying a mutation.

2. Frasersyndrome is not necessarily fatal. The lethal presentation is usually characterized by laryngeal malformations.

3. Diagnosis is mainly based on clinical findings that can be grouped as criteria.

4. Diagnosis is confirmed by genetic tests for FRAS1, FREM2 and GRIP1 gene mutations.

5. The main features of the newborn with FS are syndactyly of the fingers and toes, cryptophthalmos, abnormalities of genital organs and presence of the sibling with the same disorder.

modules in complex malformation syndromes. J Med Genet 39: 623-633.

2 Van Haelst MM, Scambler PJ, Hennekam RCM, Fraser Syndrome Collaboration Group (2007) Fraser syndrome: A clinical study of 59 
cases and evaluation of diagnostic criteria. Am J Med Genet A 143A: 3194-3203.

3 Agashe AP, Adrianwala SD, Bhatti SS, Contractor CP (1992) Fraser's syndrome. J Postgrad Med 38: 209-210.

4 Kaplana Kumari MK, Kamath S, Mysorekar VV, Nandini G (2008) Fraser syndrome. Indian J Pathol Microbiol 51: 228-229.

5 McGregor L, Makela V, Darling SM, Vrontou S, Chalepakis G, et al. (2003) Fraser syndrome and mouse blebbing phenotype caused by mutations in FRAS1/Fras1 encoding a putative extracellular matrix protein. Nat Genet 34: 203-208.

6 Vogel MJ, Van Zon P, Brueton L, Gijzen M, Cox P, et al. (2012) Mutations in GRIP1 cause Fraser syndrome. J Med Genet 49: 303-306.

7 Hoefele J, Wilhelm C, Schiesser M, Mack R, Heinrich U, et al. (2013) Expanding the mutation spectrum for Fraser syndrome: Identification of a novel heterozygous deletion in FRAS1. Gene 520: 194-197.

8 Maruotti GM, Paladini D, Agangi A, Martinelli P (2004) Prospective prenatal diagnosis of Fraser syndrome variant in a family with negative history. Prenat Diagn 24: 69-70. 